\title{
WIND ENERGY CONVERTERS AND PHOTOVOLTAICS FOR GENERATION OF ELECTRICITY AFTER NATURAL DISASTERS
}

\author{
JON OLAUSON $^{1,2}$, ANDERS GOUDE $^{1}$ and MIKAEL BERGKVIST ${ }^{1,2}$
}

${ }^{1}$ Division of Electricity, Department of Engineering Sciences, Uppsala University, Uppsala, Sweden
${ }^{2}$ Centre for Natural Disaster Science (CNDS), Uppsala, Sweden

Olauson, J., Goude, A. and Bergkvist, M., 2014. Wind energy converters and photovoltaics for generation of electricity after natural disasters. Geografiska Annaler: Series A, Physical Geography, ••, ••-••. DoI:10.1111/geoa.12052

ABSTRACT. During recovery and reconstruction after a natural disaster, an autonomous power supply might be needed for an extended period of time. In this work, the feasibility of using small-scale wind power and battery storage for power supply is evaluated and compared with systems containing photovoltaics. The investment cost per yearly produced $\mathrm{kWh}$ and for an optimized energy system supplying small loads ( 2 or $20 \mathrm{~kW}$ peak) has been calculated for 32 sites, predominantly in Africa and the Middle East. The sites represent foreign activities of the Swedish Civil Contingencies Agency at the end of 2012.

Since wind speed measurement series often have a lot of missing data, autoregressive moving average models were trained and used to generate hourly time series of wind speed. This methodology proved robust, even when data availability was very low or when measurements were only taken every third hour. The results of the simulations show that photovoltaic/battery systems outperform wind/battery systems at all evaluated sites. This can be explained by lower investment cost per yearly produced $\mathrm{kWh}$ and smoother daily/weekly power output over the year for the photovoltaic system. The proportion of wind power for optimized systems comprising wind, photovoltaics and battery bank is generally very low and the system cost is almost identical to the corresponding photovoltaic/battery systems. In conclusion, at lower latitudes and with little time for a proper wind measurement campaign, photovoltaics should be the primary candidate for replacing or complementing conventional diesel generators.

Key words: small-scale wind power, wind energy converter, photovoltaic, hybrid energy system, natural disaster

\section{Introduction}

Natural disasters often disturb the regular power system and an emergency power supply is necessary in the immediate response. If the natural dis- aster affects more remote areas, a portable and flexible power supply might be needed for an extended time period during recovery and reconstruction. One of the work packages in the research school Centre for Natural Disaster Science $(C N D S)$ thus deals with autonomous and robust information systems and energy management in relation to natural disasters (CNDS, Science plan for the Centre for Natural Disaster Science (CNDS), http://www.cnds.se/, 28 Jan., 2014).

The objective of this work is to evaluate the economic feasibility of using wind energy converters (WECs) and a battery storage for off-grid applications. Because of the unpredictable geographical location of natural disasters, all of the Swedish Civil Contingencies Agency (MSB) foreign activities as of August 2012 are chosen as study cases. This methodology contrasts with that used by most studies of small-scale wind power (hybrid) systems, where only one, often very windy, site is analysed. For comparison, photovoltaic $(P V) /$ battery and wind/PV/battery systems are also studied. Since measured time series of wind speed often have lot of missing data autoregressive moving average (ARMA) models are trained and used to generate complete time series.

In rural areas without access to the main electrical grid, power is often produced locally by diesel generators. Due to high fuel prices, difficulties with fuel transport and environmental concerns there has been substantial interest in alternative solutions in the past decades, one being hybrid systems comprising combinations of, for example, wind power, $\mathrm{PV}$, conventional diesel generators and battery storage. One of the underlying motives for the studies is that wind and solar often have complementary characteristics and thus a hybrid system 


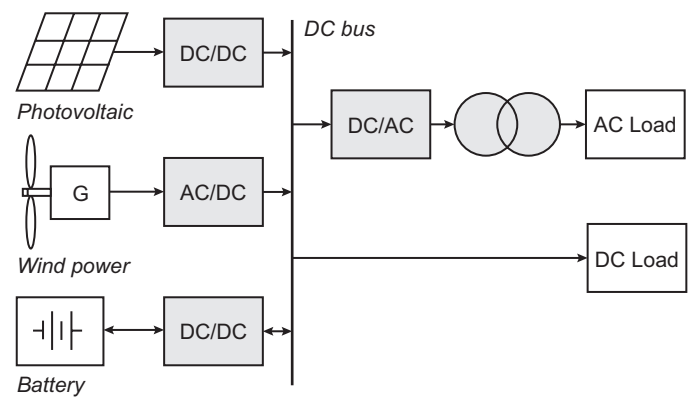

Fig. 1. Example of a wind/PV/battery hybrid system. Adapted from Xu et al. (2013).

comprising the two sources can have a more smooth power output, higher reliability and lower overall cost. Relatively recent reviews of methods and models used when optimizing a hybrid system can be found in Nema et al. (2009) and Zhou et al. (2010). A systematic but older review together with operational guidelines, an overview of operating hybrid systems etc. is found in a series of reports from Ris $\varnothing$ (Lundsager et al. 2001 and sub-reports). An example of a hybrid system topology without a diesel generator is shown in Fig. 1.

Today's state-of-the-art hybrid power systems have benefited from development in several fields, including power electronics, improvement of manufacturing techniques and efficiency of PV modules, simulation software, automatic controllers and deep-cycle lead-acid battery technology (Nema et al. 2009).

According to Zhou et al. (2010), simulations of hybrid systems can be based on either (hourly) time series of local meteorological data, on synthetic time series generated from, for example, monthly averages or on extrapolation from a nearby site. Several different techniques also exist to optimize the hybrid system with regards to high reliability, low cost, low emissions and favourable operation of batteries and diesel generators. Some examples are linear programming, probabilistic approaches, iterative techniques, dynamic programming and genetic algorithms.

Bagen and Billinton (2005) used a relatively simple model of a hybrid system containing wind and/or solar cells, diesel generators and possibly battery storage. They showed that adding battery storage significantly improves system reliability, diesel fuel savings and (most predominantly) the number of required start/stop cycles of the diesel generators. For the same installed power, wind turbines performed slightly better than solar cells. As in Billinton et al. (2003), an ARMA model was used to generate time series of wind speed. An interesting result is that there is a relatively large spread in the measures evaluated when using single year time series drawn from the 6000 simulated years.

Xu et al. (2013) built a more detailed model of the system and used a multi-objective algorithm to optimize system cost, battery state, power quality etc. No diesel generator was included and both off-grid and grid-connected operation was considered. Ashok (2007) evaluated hybrid systems containing different combinations of small-scale hydro, wind and PV together with a diesel generator and battery storage. The site had very favourable wind conditions and it was concluded that a combination of hydro and wind power offered the lowest cost and had the potential to supply a remote village in India with electricity more securely and at a lower cost compared with the diesel generator currently used.

Yang et al. (2008) used a genetic algorithm to cost optimize a system with wind power, PV and battery storage given an allowed loss of power supply probability. They included, among other parameters, PV slope angle and height of the wind turbine tower. Some conclusions were that a slightly steeper angle than optimum angle for total annual energy production was better in a hybrid system context and that a battery storage equivalent to 3-5 days of system load was optimal. Tina et al. (2006) showed that a probabilistic approach based on the probability density functions of wind power and PV output can give similar results to the more computationally and data demanding time series techniques normally used.

\section{Data}

To study the feasibility of using small-scale wind power, possibly in a hybrid system with PV, for autonomous generation of electricity, all locations of MSB foreign activities as of August 2012 were chosen as study cases. Most of the 32 sites are located in Africa or the Middle East, but the selection also includes one site each in Afghanistan, Haiti, Georgia and Kosovo.

\section{Wind speed data}

Wind speed data between 2005 and 2011 from surface measurement stations were gathered if the distance to the site was less than $10 \mathrm{~km}$. Data of 
shifting quality were found for 26 out of 32 sites, all of which were measured in stations included in the World Meteorological Organization (WMO) network. According to the WMO guidelines (WMO 2012), surface wind speed is to be measured $10 \mathrm{~m}$ above ground, reported as $10 \mathrm{~min}$ averages and the measurement should be representative of the area and not disturbed by local obstacles, such as trees or buildings. If the measurement height differs from $10 \mathrm{~m}$, a transformation method is given in the guidelines.

Although WMO claims that all measurements in the WMO network are performed according to the standard (Eichler, K., WMO/WCP, pers. com., 6 Dec., 2013), it is known from earlier experience that this is not always the case. For instance, a few measurements in Sweden are taken at $6 \mathrm{~m}$ above ground without any proper transformation (Hellström, S., SMHI, pers. com., 14 Dec., 2013). This is also one of the reasons why ECMWF does not assimilate wind measurements from land surface stations in their weather model (Garcon, H., ECMWF, pers. com., 16 Dec., 2013). Contacts have been taken with the national meteorological institutes responsible for the measurements. Those who replied all confirmed $10 \mathrm{~m}$ as the measurement height.

Because of the lack of data for six sites and the possibility that a few wind measurements are performed at a height differing from $10 \mathrm{~m}$, it was decided to also include time series from a meteorological model in the analysis. Reanalysis data from Modern Era Retrospective Analysis (MERRA) (Lucchesi, R., File specification for MERRA products. GMAO Office Note No. 1 (Version 2.3), http:// gmao.gsfc.nasa.gov/pubs/office_notes, 20 Jun., 2014), was chosen because of the relatively high temporal resolution $(1 \mathrm{~h})$, its global coverage and its free availability. Wind speeds in the model are given at $10 \mathrm{~m}$ above displacement height and $50 \mathrm{~m}$ above ground. It must be stressed that the model resolution is $1 / 2^{\circ} \times 2 / 3^{\circ}$, which implies that local effects on the wind speed are not captured properly. However, the main focus of this work is not to assess the performance of wind power at particular sites but rather the general feasibility in areas where the proposed solution might be applied.

\section{Solar irradiation data}

One year of solar irradiation data from the HelioClim-3 model for an optimal inclined plane was retrieved. The model is based on satellite measurements and has shown good agreement with measurements: 'several studies demonstrate the superiority of the use of such data compared to interpolation methods applied to spatially and temporally sparse measurements performed within a pyranometric network' (Espinar et al. 2012). Two of the MSB sites were slightly outside the modelled area in HelioClim. In these cases data from the same latitude but within the model area were used. The replacement sites were chosen to give the same yearly insolation according to the MERRA model.

\section{Components in hybrid system}

Certified power curves from two commercial WECs were used in the simulations, one for a small system (maximum load of $2 \mathrm{~kW}$ ) and one for a medium-sized system (maximum load of $20 \mathrm{~kW}$ ). The WEC chosen for the small system is manufactured by Bergey and has a rated power of $10 \mathrm{~kW}$ at $11.5 \mathrm{~m} \mathrm{~s}^{-1}$, although it produces up to $12.5 \mathrm{~kW}$ at higher wind speeds. Maximum overall efficiency is $30 \%$ and rated power per swept rotor area is $260 \mathrm{~W} \mathrm{~m}^{-2}$. The larger WEC is from Northern Power and has $60 \mathrm{~kW}$ rated power. It is an extreme low wind turbine $\left(144 \mathrm{~W} \mathrm{~m}^{-2}\right)$ and also has high efficiency, up to $39 \%$, which results in a capacity factor around 40-110\% higher compared with Bergey at the same wind conditions. Both turbines are of the three-bladed horizontal axis type. Twenty meters hub height is assumed for the smaller turbine and $40 \mathrm{~m}$ for the larger. Power curves for the two WECs are given in Fig. 2.

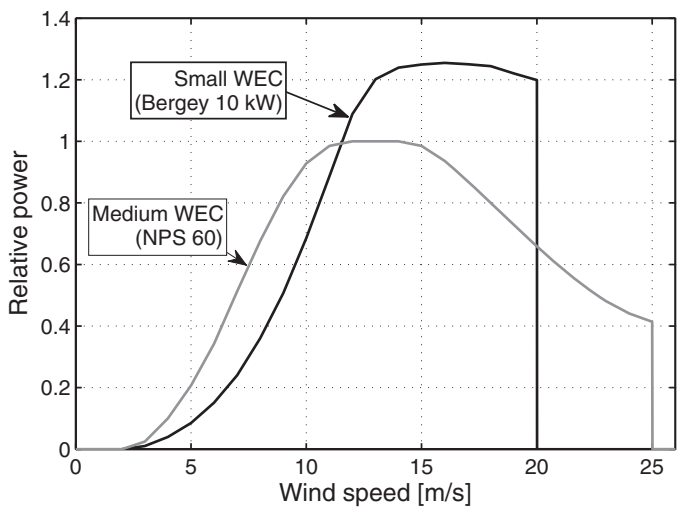

Fig. 2. Power curves for the two WECs used in the simulations. The power is given relative to rated power $(10 \mathrm{~kW}$ and $60 \mathrm{~kW}$ respectively). 
Based on completed projects, RenewableUK found costs of $£ 4-4.9 \mathrm{~W}^{-1}$ for WEC in the range $5-50 \mathrm{~kW}$ and $£ 3.5 \mathrm{~W}^{-1}$ for $100 \mathrm{~kW} W E C$. It should be noted that costs have increased in the last few years, which can partly be explained by increasing metal prices. The expectations for cost reductions in the short term are moderate (RenewableUK, Small and medium wind market report 2012, http:// www.renewableuk.com/, 14 Dec., 2013). The average capacity-weighted installed cost of small wind turbines sold in the USA in 2012 was $\$ 6.5 \mathrm{~W}^{-1}$ (US Department of Energy, 2012 Market report on U.S. wind technologies in distributed applications, http://www1.eere.energy.gov/wind/, 3 Jan., 2014). The assumed cost for both WECs used in the simulations is $€ 4.8 \mathrm{~W}^{-1}$.

Contrary to the situation for small-scale wind power, there have been dramatic reductions in cost of PV projects over the last years. A report from IEA (IEA-PVPS, Trends 2013 in photovoltaic application, http://www.iea-pvps.org/, 3 Jan., 2014) lists indicative prices for installed PV systems in selected countries in 2012, given as cost per nominal power (power at standard test conditions: $\mathrm{W}_{\mathrm{p}}$ ). For grid-connected systems below $10 \mathrm{~kW}_{\mathrm{p}}$, figures between $€ 1.3 \mathrm{~W}_{\mathrm{p}}^{-1}$ and $€ 5.0 \mathrm{~W}_{\mathrm{p}}^{-1}$ are reported while larger systems cost $€ 1.0-4.7 \mathrm{~W}_{\mathrm{p}}^{-1}$. Swedish figures, $€ 2.4 \mathrm{~W}_{\mathrm{p}}^{-1}$ for the small systems and $€ 1.8 \mathrm{~W}_{\mathrm{p}}{ }^{-1}$ for medium systems, were used in the simulations. These costs are well in line with the average for all studied countries.

Based on Zhou et al. (2011), Pickard (2012) and $\mathrm{Xu}$ et al. (2013) it is reasonable to estimate the cost of lead acid batteries at $€ 100 \mathrm{~kW}^{-1} \mathrm{~h}^{-1}$. Cables, control system, converters etc. are assumed to be included in the cost for WEC and PV, since these figures are given for complete projects.

\section{Method \\ ARMA}

A natural approach to produce time series without missing data would be measure-correlate-predict (MCP), see e.g. Thøgersen et al. (2007). MCP can be seen as a simple form of statistical downscaling of the results from a meteorological model (MERRA in this case). A few different MCP techniques were evaluated, including linear regression with different types of residual resampling. Although mean wind speed and energy yield can be well reproduced with these methods, the temporal characteristics of the wind speed and therefore the cost of an optimized wind/battery system proved very sensitive and hard to correctly capture. Because of this it was decided to evaluate the use of ARMA models.

ARMA models and extensions of this concept are popular time series tools used in various different fields. ARMA models have been used to model wind speed and wind power for a long time, both for short-term forecasting (Shi et al. 2011; Pinson and Madsen 2012) and for generation of arbitrary long fictive time series for power system analysis etc. A simulation of a process by an autoregressive model recognizes that a time series is not only characterised by its probability density function (approximately Weibull for wind speed), but also by the persistency of the process, or in other words, how the process correlates with the same process shifted in time. The latter is described by the autocorrelation function $(A C F)$. Of interest is also the partial ACF (PACF), which describes the correlation between adjacent observations $y_{t}$ and $y_{t-k}$ when the mutual linear dependencies of $y_{t-1} \ldots y_{t-k+1}$ are removed. The sample ACF at lag $k$ of an observed time series $y_{l} \ldots y_{n}$ can be calculated by:

$$
A C F_{k}=\frac{\sum_{t=k+1}^{n}\left(y_{t}-\bar{y}\right)\left(y_{t-k}-\bar{y}\right)}{\sum_{t=1}^{n}\left(y_{t}-\bar{y}\right)^{2}} .
$$

The autocorrelation of the wind speed is of great importance for off-grid wind power since long periods of low wind speeds reduce reliability or increase the demand for storage systems or auxiliary generation, such as diesel generators.

An ARMA process with AR order $p$ and MA order $q$ is notated $\operatorname{ARMA}(p, q)$ and satisfies the equation:

$$
\begin{aligned}
Y_{t}= & \phi_{1} Y_{t-1}+\phi_{2} Y_{t-2}+\ldots+\phi_{p} Y_{t-p}+e_{t} \\
& -\theta_{1} e_{t-1}-\theta_{2} e_{t-2}-\ldots-\theta_{q} e_{t-q}
\end{aligned}
$$

where $\phi_{i}$ are AR coefficients, $\theta_{j}$ are MA coefficients and $e$ is Gaussian white noise with mean 0 and variance $\sigma^{2}$. Following the steps of Box and Jenkins as referred to in Lojowska et al. (2010), a proper ARMA model could be found:

1. Transform the original data to stationarity (loosely meaning that mean and variance are constant in time) and normality.

2. Identify suitable model candidates by studying the transformed time series and the ACF and PACF thereof. 
3. Estimate model parameters. This can be done using, e.g., least square or maximum likelihood estimation (Cryer and Chan 2008).

4. Evaluate the model and, if necessary, repeat steps 2-4.

Several transform methods have been used in the wind power literature. McWilliams and Sprevak (1982) divided the wind speed into two orthogonal components, one in the prevailing wind direction, and argued that these series should be normally distributed and independent and ready for AR simulation. Chen et al. (2010) used a Box-Cox transformation while Lojowska et al. (2010) found the Weibull CDF corresponding to each hour in each month and transformed the data to normality. A similar approach as in Billinton et al. (1996) and $\mathrm{Li}$ and Wang (2012) was used in this work to transform the wind speed time series $u(t)$ to stationarity:

$$
y_{0}(t)=\frac{u(t)-\mu(m, h)}{\sigma(m, h)},
$$

where $\mu$ and $\sigma$ are sample mean and standard deviation for each hour in each month. In addition, the resulting time series were transformed to approximate normality with the Box-Cox transformation

$$
y(t)=\frac{y_{0}(t)^{\lambda}-1}{\lambda} .
$$

The lambda parameter was optimized for each individual site using maximum likelihood estimation. Finally, a transformation to $N(0,1)$ was performed. The transformed time series are very close to normality within around \pm 2.5 standard deviations, but have heavy tails.

The models were evaluated using Aikake's information criteria (AIC), by studying the distribution and ACF of the residuals, by comparing model and original time series ACF, and by visual inspection of time series generated by the model. The cost of a wind/battery system using ARMAgenerated wind speed and measured wind data from stations with good availability were also compared.

To estimate the parameters of the ARMA models, various software can be used. The Matlab $2012 \mathrm{~b}$ optimizer was found to be robust and give good results, even without initial parameter guesses. Missing data are however just removed, which give erroneous results for series with sub- stantial lack of data. The statistical software $\mathrm{R}$ (The R Core Team, $R$ : a language and environment for statistical computing, reference index version 3.0.2, http://www.r-project.org/, 20 Jun., 2014; Hyndman, R.J., et al., Forecast: forecasting functions for time series and linear models, http:// CRAN.R-project.org/package=forecast, 20 Jun., 2014), however, can properly handle missing data but sometimes fails to converge or to find globally optimized parameters if no proper initial guesses of parameter values are supplied. Because of this, Matlab was used to find initial parameter estimates, which were then fed to $\mathrm{R}$ for final determination. Maximum likelihood estimation was employed in both cases.

\section{Modelling of wind power}

To estimate the wind speed at other heights than measured, the power law can be used:

$$
u(z)=u\left(z_{\text {ref }}\right)\left(\frac{z-d}{z_{\text {ref }}-d}\right)^{\alpha},
$$

where $z$ is height above ground, $d$ is displacement height and $\alpha$ the power law exponent. The displacement height is a variable depending on, for example, canopy height and density, typically $d$ is in the range from $2 / 3$ to 1 of canopy height. If measurements are performed at two or more heights, $\alpha$ can be calculated directly for each individual time step, otherwise an estimation is needed. An often used method is to use a constant $\alpha$ by parameterizing $\alpha$ as a function of mean wind speed and/or surface roughness. A disadvantage of this method, in particular if time-dependent energy production is of interest, is that the often substantial temporal variations of $\alpha$ are neglected. Therefore the following method was used instead:

1. Mean $\alpha$ was estimated as a function of mean wind speed according to the observed relation of $\alpha$ and mean wind speed in the MERRA model (for the 32 sites evaluated). This gave $\alpha$ estimates of 0.25 for $10 \mathrm{~m}$ mean wind speed of $2 \mathrm{~m} / \mathrm{s}$ down to 0.13 for $6 \mathrm{~m} \mathrm{~s}^{-1}$.

2. The time-varying characteristics of $\alpha$ were taken from the bi-linearly interpolated MERRA time series at the site.

Hourly energy generated from the WEC was calculated based on measured power curves for two commercial turbines. Ten percent additional losses 

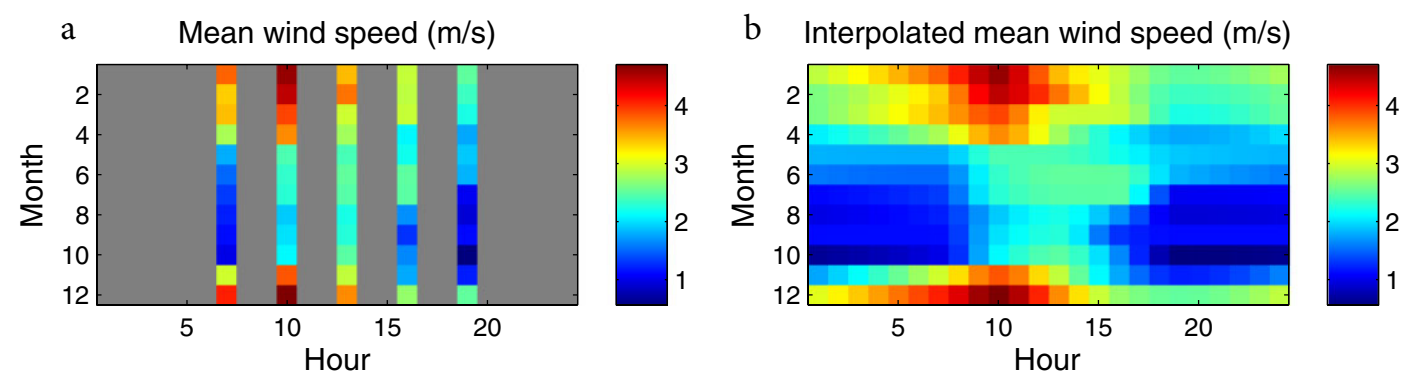

Fig. 3. Example of interpolation. (a) Original mean wind speed. For this station measurements are only taken during daytime every third hour. (b) Interpolated mean wind speed using Matlab shape-preserving piecewise cubic Hermite interpolation for each month.

due to park effects, dirt on blades, maintenance, forced outages etc. was assumed. The inclusion of forced outages in the general losses is a simplification; a system consisting of only one WEC and batteries would have higher loss of load probability $(L O L P)$ if forced outages were simulated as discrete events and not "smoothened out" over the whole year. Since the vast majority of the optimized wind/battery systems contain at least four WECs, this effect is thought to be very small.

As is explained further in the results section, not all of the wind measurements were found appropriate for direct ARMA modelling. For these stations the following methodology was used instead:

1. Mean wind speeds and standard deviations were interpolated to fill months/hours with no data. Matlab shape-preserving piecewise cubic Hermite interpolation was used for each month (see Fig. 3).

2. Based on the ACF function, the most similar of the produced ARMA processes was chosen to generate transformed data $(y)$. To find the most similar ACF, a weighting function was used so that the match of the first lags of the ACF was given higher priority.

3. Back transformation $(y \rightarrow u)$ was performed using (interpolated) mean wind, standard deviation and Box-Cox parameter from the original time series.

As is shown in the results section, this approach proved very successful.

\section{Modelling of $P V$ power}

The model for hourly energy generated from PV is adopted from Huld et al. (2010). The output power is a function of in-plane irradiance $G$ and module temperature $T_{\text {mod }}$

$$
P\left(G, T_{m o d}\right)=P_{S T C} \cdot \frac{G}{G_{S T C}} \cdot \eta_{r e l}\left(G^{\prime}, T^{\prime}\right),
$$

where $\eta_{r e l}$ is instantaneous relative efficiency. Subscript STC indicates standard test conditions and prim values are normalized to STC: $G^{\prime}=G / G_{S T C}$ and $T^{\prime}=T_{\text {mod }}-T_{\text {mod,STC }}$ where $G_{S T C}=1000 \mathrm{~W} / \mathrm{m}^{2}$ and $T_{\text {mod,STC }}=25^{\circ} \mathrm{C}$. Instantaneous relative efficiency is given by:

$$
\begin{aligned}
\eta_{r e l}\left(G^{\prime}, T^{\prime}\right)= & 1+k_{1} \ln G^{\prime}+k_{2}\left(\ln G^{\prime}\right)^{2} \\
& +T^{\prime} \cdot\left[k_{3}+k_{4} \ln G^{\prime}+k_{5}\left(\ln G^{\prime}\right)^{2}\right] \\
& +k_{6} T^{\prime 2}
\end{aligned}
$$

$T_{\text {mod }}$ can be estimated from ambient temperature and irradiance by:

$$
T_{m o d}=T_{a m b}+c_{T} G
$$

The constants $k_{I}-k_{6}$ and $c_{T}$ can be fitted to measured data for a certain PV configuration. Assuming crystalline silicon $\mathrm{PV}$, the following constants were adopted from Huld et al. (2010): $k_{1}=-0.017162$, $k_{2}=-0.040289, \quad k_{3}=-0.004681, \quad k_{4}=0.000148$, $k_{5}=0.000169, k_{6}=0.000005, c_{T}=0.035$.

In addition to the efficiency given by $\eta_{\text {rel }}, 3 \%$ reflectance losses and 14\% losses due to dirt, cables, converters etc. were added. For the sites evaluated, the method gave a total average system efficiency ranging from $70 \%$ to $79 \%$.

\section{Modelling of hybrid system}

A relatively simple model of the hybrid system was used, based on hourly energy generated from the WEC and/or PV system, energy consumed by the load and inflow/outflow of energy in the battery bank. Voltage levels, varying internal resistance in the batteries, system stability and control etc. were 
not considered. The IEEE Reliability Test System load profile was used ( 2 and $20 \mathrm{~kW}$ peak load) with summer peak as default (Albrecht et al. 1979). The load factor is 0.61 , corresponding to an annual consumption of 10,800 and $108,000 \mathrm{kWh}$, respectively. Since irradiance and wind speed are given in UTC time, a proper time shift was applied to the load curve.

Whenever the hourly generated electricity exceeds the load, the battery is charged. If the opposite occurs the battery assists in supplying the load. However, the battery is not allowed to go below the rated depth of discharge or above full charge. Round-trip efficiency is assumed to be $85 \%$. The hours when generation and batteries fail to supply the load are counted and the LOLP, i.e. the proportion of time when load is not supplied, is calculated.

\section{Optimization procedure}

The optimization problem to be solved was to find components to minimize system costs, given an allowed LOLP. For nonlinear optimization problems, such as the one present in the current work, there is a possibility that the cost surface has several local minima, which can cause local optimization algorithms to not find the globally best minimum. Therefore, the method of choice in the current work was to perform the optimization in two steps. The initial step used the global optimization method "lowest energy pivot method"
(Stanton et al. 1997), which randomly distributes a number of points throughout the parameter space and keeps moving the points with the highest energy towards the points with lower energy until the minimum is found. After a sufficient number of steps with this method to find the correct local minimum, the point with the lowest energy was chosen and a Broyden-Fletcher-Goldfarb-Shanno method (Shanno 1970), which is a quasi-Newton local optimizer, was used to quickly find the optimal point.

The installed power/storage capacity of WEC, PV and battery bank are varied smoothly in the optimization procedure, i.e. it is assumed that any desired power and energy storage levels could be accomplished.

\section{Results}

This section contain results for the ARMA modelling and hybrid system simulations. The locations of the 32 studied sites are shown in Fig. 4 and some basic data for the sites are presented in Table 1. The measured mean wind speed is given as weighted mean of monthly/hourly means. The reason for this approach is that several measurements have an irregular pattern, e.g. fewer measurements during night-time. MERRA mean wind speed is given for $10 \mathrm{~m}$ above displacement height. Average solar irradiation is given for the optimal fixed plane regarding annual energy production. One column also indicates whether the measured wind speed

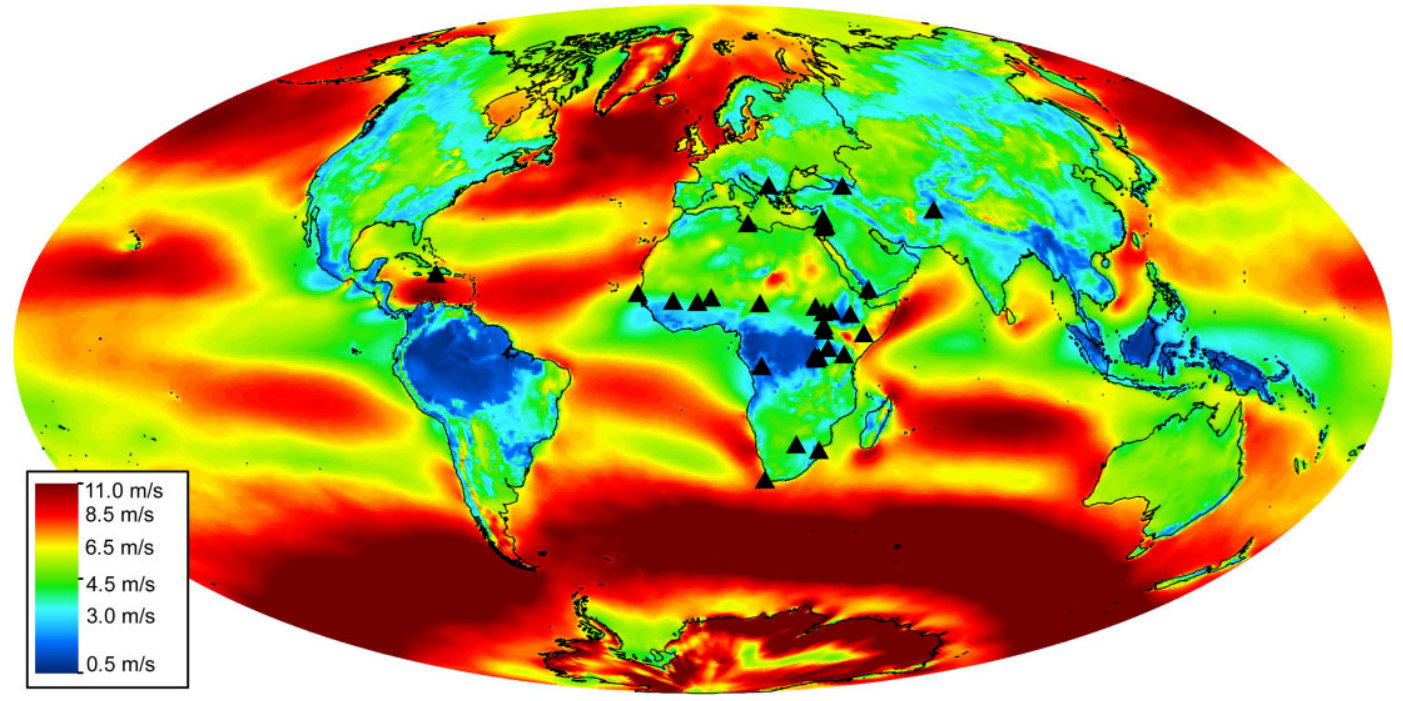

Fig. 4. Site locations and mean wind speed $10 \mathrm{~m}$ above displacement height (MERRA for year 2010). 


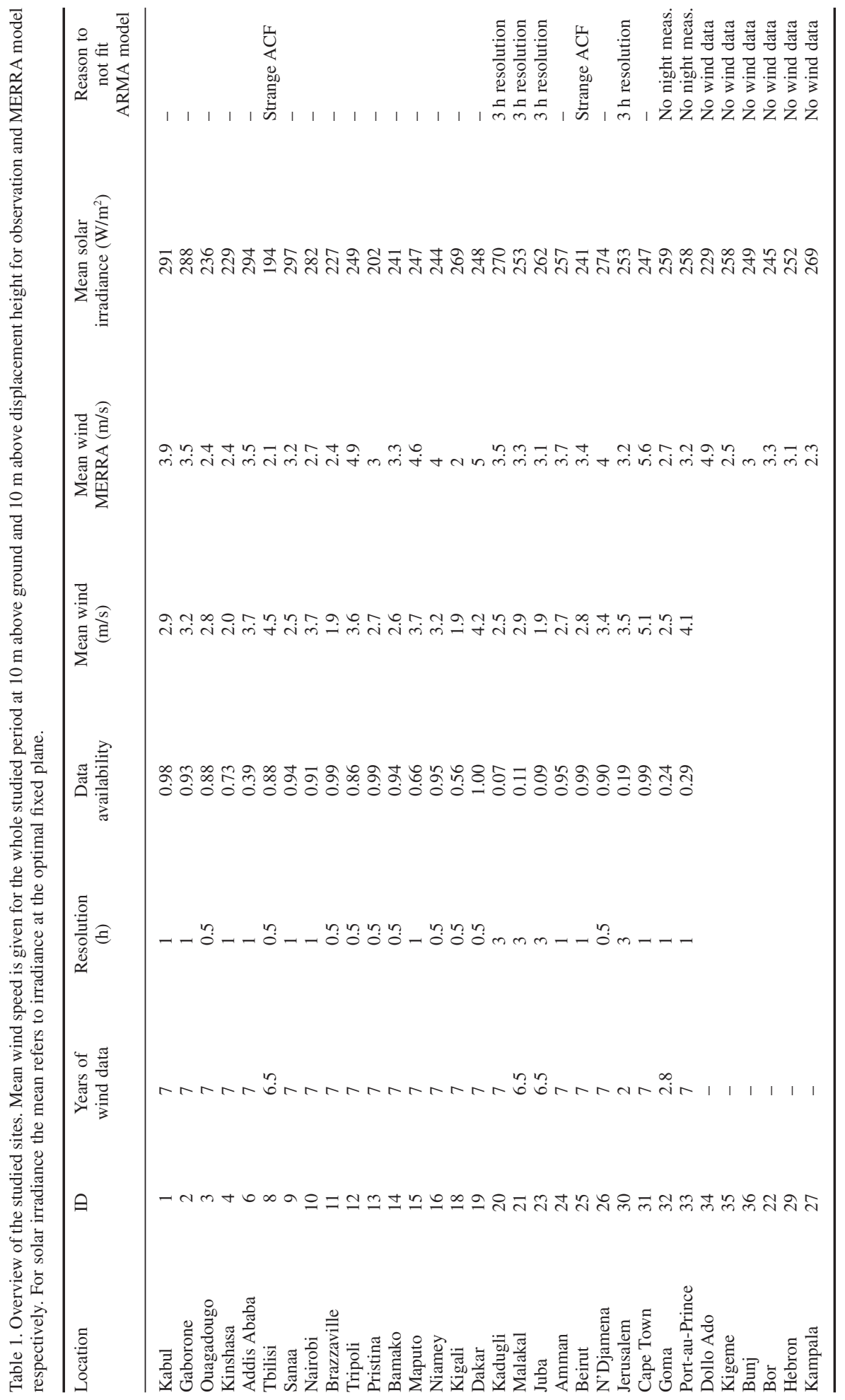




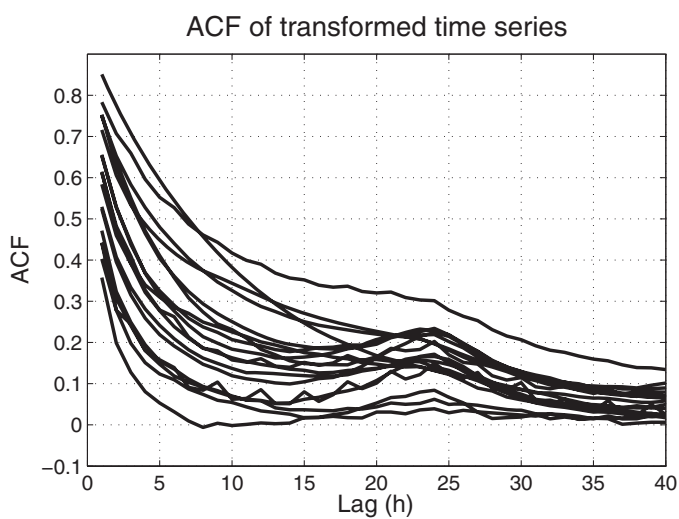

Fig. 5. ACF of the 18 transformed time series. Most ACF have a diurnal peak.

time series have been judged satisfactory for ARMA modelling. The main reason for not fitting an ARMA model is that several measurements have a temporal resolution of $3 \mathrm{~h}$; in other cases data are not recorded during night-time or the measurements show disturbing patterns that are thought to be disinformative in the ARMA modelling. In total, 18 time series were considered appropriate for modelling.

\section{ARMA modelling}

Previous research (Billinton et al. 1996) has showed that an ARMA $(4,3)$ process can be a suitable candidate to model the transformed wind speed time series. It was validated that this process can capture the ACF and PACF of the studied time series reasonably well. However, for most of the sites there is a substantial increase in the ACF of the transformed time series around $24 \mathrm{~h}$ lag (see Fig. 5), which resulted in significant ACF of the model residuals around the same lag. One solution would have been to include a seasonal component in the process by using $\operatorname{ARMA}(4,3) \times(\mathrm{P}, \mathrm{Q})_{24}$ (Cryer and Chan 2008). However, better results were achieved by using an $\operatorname{ARMA}(24,3)$ process with AR coefficients 5-20 set to zero. For clarity, this process is denoted $\operatorname{ARMA}(24 * 3)$. Full AR(24) and $\operatorname{ARMA}(24,3)$ models were also found suitable for some time series.

It is interesting to note that in the literature of autoregressive models in the wind power field, relatively low-order models have been found sufficient. A model with few parameters is always preferable if it can satisfactorily capture the char-

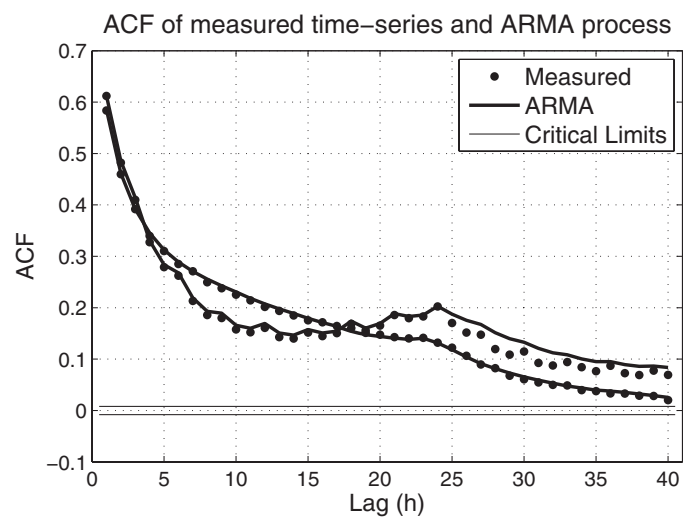

Fig. 6. Two examples, one relatively good and one relatively bad, of the match of ACF of transformed observed wind speed and corresponding ARMA process.

acteristics of the modelled process. This does not seem to be the case for most of the measurements studied in this work. A likely explanation is that, using data from the MERRA model, the transformed wind speed time series in Africa/Middle East have a much more pronounced ACF increase around $24 \mathrm{~h}$ lag compared with those in Europe and North America.

Although not all possible combinations of ARMA process orders were evaluated for all 18 time series, AIC calculations indicated the appropriateness of the four candidates over neighbouring models, e.g. ARMA $(3,2)$ or $\operatorname{ARMA}(5,4)$. Parameters for the four selected models were estimated for all 18 time series, and primarily based on the AIC criteria, the most suitable were selected for each site. The match of ACF and ACF of residuals was also visually inspected and for two sites overrode the selection of model based on lowest AIC. Most time series were best modelled by the $\operatorname{ARMA}\left(24^{*}, 3\right)$ or $\operatorname{ARMA}(24,3)$ process, but $\operatorname{ARMA}(4,3)$ and $\operatorname{AR}(24)$ were also selected for a few sites. Two examples of ACF for the observed time series and the estimated ARMA processes are found in Fig. 6. In Fig. 7, the hourly mean wind speed for an observed and simulated time series is shown (for January and July, respectively). Figure 8 shows an example of an original and simulated wind speed time series. Note that the two series are not supposed to be very well correlated, but rather show a similar standard deviation, autocorrelation, diurnal pattern etc.

Since many of the studied time series have a lot of missing data, a test was performed to evaluate the effect of the missing data on the results. A 
station with nearly $100 \%$ availability (Kabul) was used as reference and compared with the same station where data were removed according to the missing data pattern for three other stations with $94 \%, 66 \%$ and $39 \%$ availability, respectively ("good", "bad" and "ugly"). As can be seen in Fig. 9, the theoretical ACF for the resulting $\operatorname{ARMA}(24 *, 3)$ models only differs slightly. The mean wind, capacity factor and cost for optimized wind/battery systems with and without removed data are found in Table 2. It can be concluded that ARMA modelling is a robust tool when dealing with time series with a lot of missing data.

A comparison was also made between simulation results using "raw" data from seven stations with over $95 \%$ availability (by simply removing NaN:s) and using data from ARMA models. The mean wind speed and yearly energy yield were almost identical. The cost of optimized

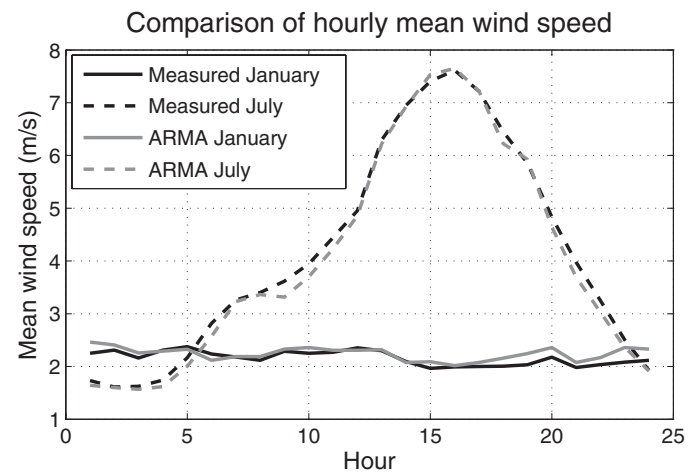

Fig. 7. Hourly mean wind speed in January and July for observed and simulated time series (Kabul station). wind/battery systems, however, differed between $8 \%$ and $17 \%$. For all stations, the cost using ARMA data was lower; bias is $11 \%$. A probable explanation is that the cost is very sensitive to the persistence of low wind speeds. Although the general ACF is captured very well by the ARMA models, real-world wind has a tendency to stay longer in those low wind regimes.

\section{New method for generation of wind speed time series}

As was explained earlier, a new method was developed to generate wind speed time series for the eight stations where fitting of an ARMA model proved difficult. The basic idea was to "borrow" an ARMA process from a station with similar ACF and then back-transform simulated data to wind speed using monthly/hourly mean wind speed and standard deviation and Box-Cox parameter from the original data.

To evaluate the feasibility of this method, two tests were performed. First, for each of the 18 stations with a fitted ARMA model, the most similar

Table 2. Effect of missing data on mean wind speed, capacity factor and cost of optimized wind/battery systems $(20 \mathrm{~kW}$ peak load).

\begin{tabular}{lccc}
\hline & $\begin{array}{c}\text { Mean wind } \\
\text { speed }(\mathrm{m} / \mathrm{s})\end{array}$ & $\begin{array}{c}\text { Capacity } \\
\text { factor }\end{array}$ & $\begin{array}{c}\text { Cost of hybrid } \\
\text { system (k€) }\end{array}$ \\
\hline All data & 3.7 & 0.15 & 1500 \\
"Good" & 3.8 & 0.15 & 1500 \\
"Bad" & 3.8 & 0.15 & 1400 \\
"Ugly" & 3.7 & 0.15 & 1400 \\
\hline
\end{tabular}

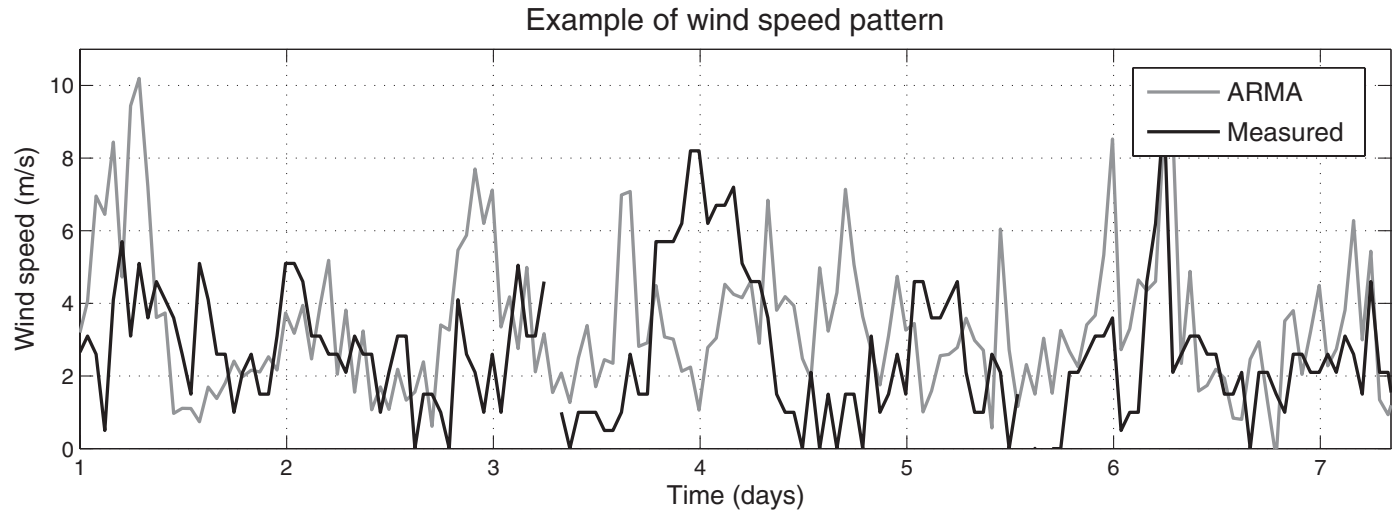

Fig. 8. Example of original and simulated wind speed time series (period with mild diurnal pattern). 


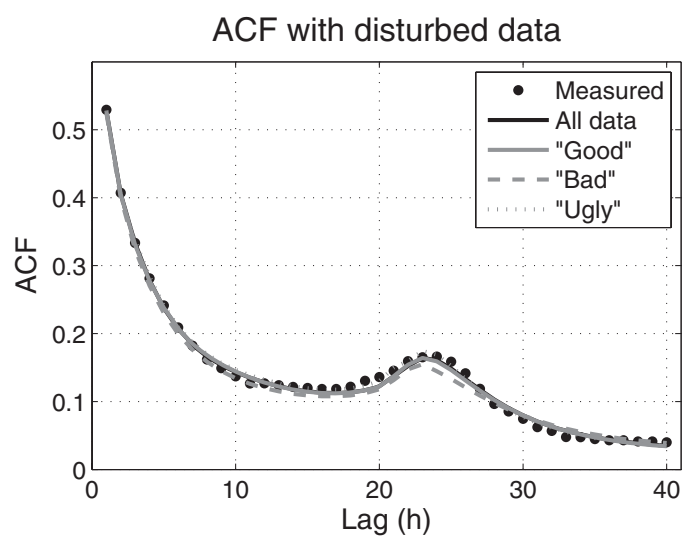

Fig. 9. Evaluation of the effect of missing data on ARMA theoretical ACF.

ARMA process regarding ACF was found and simulated data from that process were used. It was noted that processes with similar ACF also had similar variance (see Eqn 2); linear correlation of the 18 pairs of variance for the original and the "borrowed" ARMA process was 0.93. When comparing results, it was, as expected, found that mean wind speed and energy yield were almost identical. Moreover the cost of the optimized wind/battery system was very similar: correlation was 0.99 and the maximum relative difference was $9 \%$ (of which a substantial part could be explained by the use of only $7 \mathrm{yr}$ of data in the simulation).

In the second test, $94.5 \%$ of the data of the 18 stations was removed according to the missing data pattern of the station in Jerusalem and the same analysis as above was performed. Mean wind speed and energy yield as well as cost for the wind/battery system were effected to a low degree. The mean absolute error in cost estimation was $7.6 \%$, which must be considered very good given the massive amount of data removed and the sensitivity of wind/battery system cost.

\section{Annual energy yield}

In a hybrid system containing wind power, $\mathrm{PV}$ and batteries, the temporal characteristics of wind speed and solar irradiance are of great importance. When working against a deferrable load, e.g. cleaning and/or pumping water to a storage reservoir, or for grid-tied applications, the average capacity factor and the investment cost per yearly produced $\mathrm{kWh}$ is perhaps more interesting. These results are shown as boxplots in Figs 10 and 11.

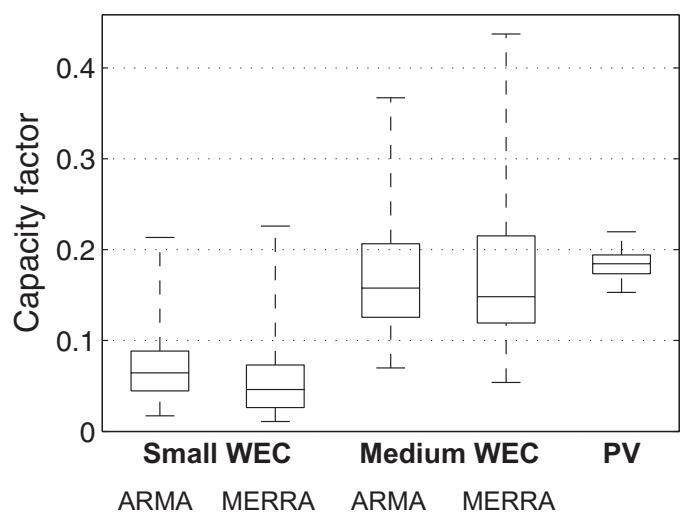

Fig. 10. Average capacity factor. On each box, the central mark is the median, the edges of the box represent the 25th and 75th percentile and whiskers extend to the most extreme data.

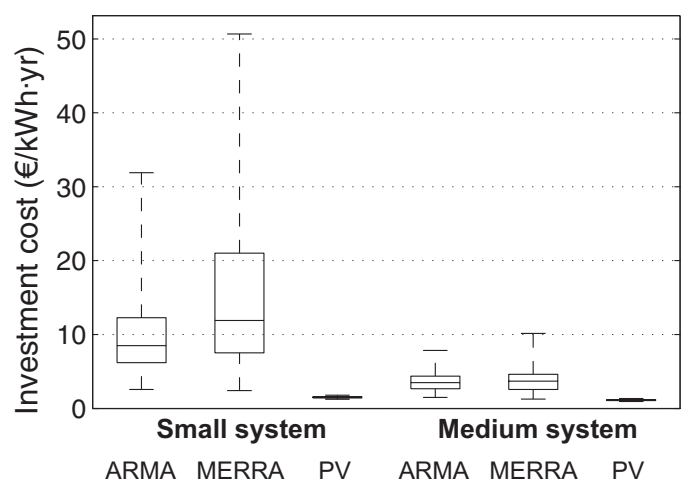

Fig. 11. Investment cost per yearly produced $k W h$. On each box, the central mark is the median, the edges of the box represent the 25 th and 75 th percentile and whiskers extend to the most extreme data.

Recall that for WEC the difference between the small and medium system lies in type of turbine and hub height, while for PV the difference lies in the investment cost per Watt.

It is obvious from the results in Fig. 11 that PV produces more electricity per invested money than wind power and that the variations between the sites are much smaller. It is also clear that a larger, more efficient, wind turbine with higher hub height performs better than a smaller one (this is of course the reason why commercial wind turbines have increased tremendously in size over the past decades). The investment cost per watt is assumed to be the same for the small and the large WEC. While it is reasonable to assume the larger WEC has a higher hub height and efficiency, it is perhaps 


\section{a}

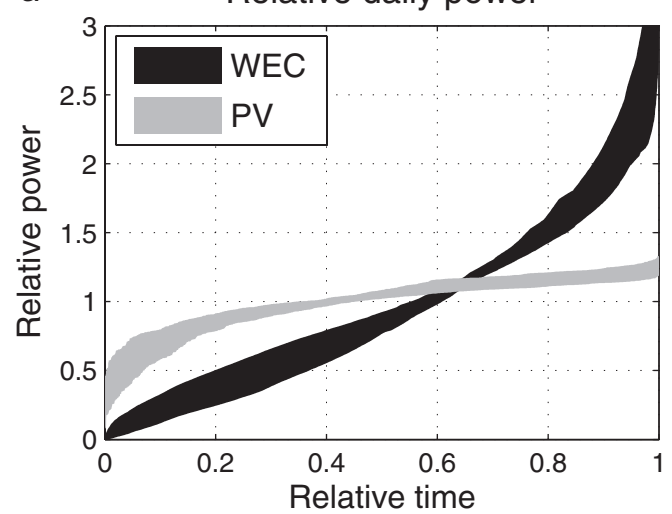

b

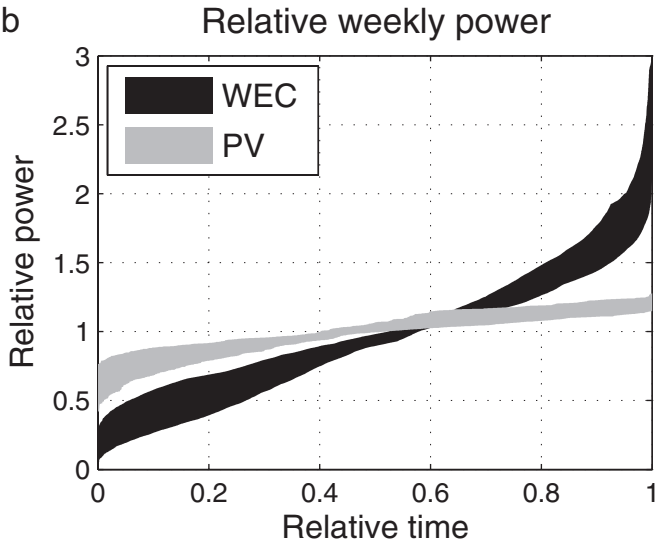

Fig. 12. Duration plot of sliding (a) 24 and (b) $168 \mathrm{~h}$ mean power relative overall mean power. $75 \%$ confidence bands out of the 32 sites are given. Wind speed is taken from ARMA simulations except for the six sites where no wind speed data are available. For these sites MERRA data are used instead.

overly optimistic to assume that the relatively big rotor (only $144 \mathrm{~W} / \mathrm{m}^{2}$ ) for the larger WEC does not come at a higher cost.

\section{Temporal characteristics of energy production}

One way to visualize the temporal characteristics of the energy production is to look at a duration plot of the sliding daily or weekly production relative to the mean production (Fig. 12). An example interpretation of the figure is that, excluding the $25 \%$ most extreme sites, the probability of mean weekly WEC production being lower than $50 \%$ of the yearly average is $6-27 \%$. For PV this probability is only $0-1 \%$. High probability for relatively low production is a strong indicator that large battery storage would be required in order to have a reliable standalone system. For the sites evaluated, PV has more favourable temporal characteristics with substantially higher low daily and weekly production relative to the average.

\section{Hybrid system}

The results presented so far strongly suggest that PV is more suitable than small-scale wind power for standalone application at the evaluated sites. As several authors have pointed out (Yang et al. 2008; Zhou et al. 2010), wind power and PV can be complementary and consequently a hybrid system can outperform single-source systems. Based on the assumptions and optimization procedure described above, the lowest costs for wind/battery,

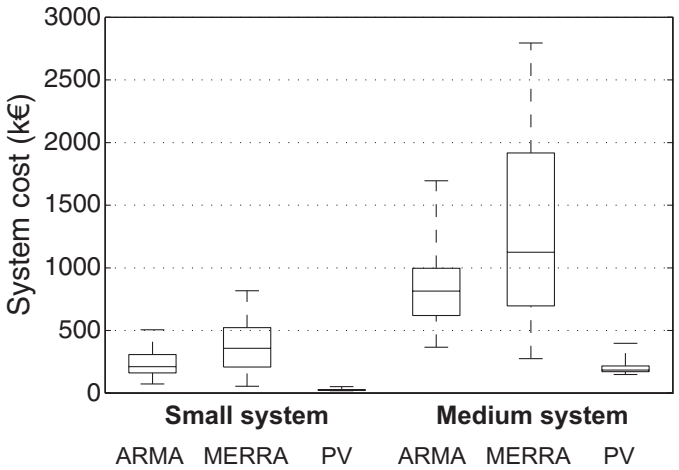

Fig. 13. Cost for optimized system with $2 \mathrm{~kW}$ and $20 \mathrm{~kW}$ peak load respectively. Only the cost for wind/battery and PV/battery systems are given since the cost for hybrid systems are almost identical to the PV/battery systems. On each box, the central mark is the median, the edges of the box represent the 25th and 75 th percentile and whiskers extend to the most extreme data.

$\mathrm{PV} /$ battery and wind/PV/battery systems were calculated for each site and for two different loads. As a default, summer peak is assumed for the load and a LOLP of $3 \%$ is accepted. The resulting costs for the small and medium systems are given in Fig. 13. The PV/battery system outperforms the wind/ battery system for both loads at all sites. In particular, for the smaller system, the cost is often an order of magnitude smaller. The cost of the optimized hybrid system is naturally always approximately equal to or lower than for the corresponding $\mathrm{PV} /$ battery system. However, the difference is very small and the optimized wind power capacity often negligible. In fact, for none of the sites the opti- 
mized wind power capacity equals the rated power of the WEC units used in the calculations. The hybrid system with the largest share of wind power is the $20 \mathrm{~kW}$ load in Tbilisi with $28 \mathrm{~kW}$ wind power, $97 \mathrm{~kW}$ PV and $461 \mathrm{kWh}$ battery capacity. For this station and load the system costs are $k € 465$ for wind $_{\mathrm{ARMA}} /$ battery, $\mathrm{k} € 398$ for PV/battery and $k € 359$ for the hybrid system.

The stations at higher latitude might be worth mentioning separately, since wind power systems are more likely to be competitive for these sites. Johannesburg is located at latitude $-34^{\circ}$, while both Tbilisi and Pristina are at latitude $42^{\circ}$. The latter two have the highest cost for PV/battery systems but Johannesburg does not have a remarkably high cost compared with the mean or median. Because of this, the cost for PV/battery is roughly half that for a wind/battery system, although the wind conditions in Johannesburg are the best for standalone systems according to both MERRA and ARMA. At Tbilisi the wind conditions are according to ARMA the second best of all evaluated sites. Combined with the highest PV/battery cost this yields, as quantified above, a relatively small difference between wind/battery and PV/battery system costs.

Generally, using wind speed data from the MERRA model gives somewhat lower capacity factors (Fig. 10) and substantially higher system costs (Fig. 13) than using ARMA data. This might seem surprising since the mean wind speed of MERRA is actually slightly higher than for ARMA. The two most dominating explanatory factors are thought to be lighter tail in the PDF for MERRA (which explains the lower average capacity factor) and less noisy data (and hence longer periods with wind speeds close to zero). A smaller part could also be attributed to the underestimation of system costs using ARMA data: $-11 \%$ bias compared with using observed wind speed.

The optimized battery capacity for systems containing PV is in the range 0.7-2.1 days (average 1.1 days) of average load and represents 12-20\% $(16 \%)$ of the system cost. For wind/battery systems, battery capacity varies from 2.5 to 16.7 days (7.0) average load and represents $12-30 \%$ (19\%) of system cost.

The obtained results are strikingly different to those in the reviewed hybrid system literature, in which hybrid solutions have often been deemed superior and wind power looks favourable in comparison to PV. Several factors have been identified explaining this difference:
1. Many studies have chosen very favourable sites regarding wind conditions; the mean wind speed is often 6-7 $\mathrm{m} \mathrm{s}^{-1}$ at low height and in one case was as high as $9.1 \mathrm{~m} \mathrm{~s}^{-1}$ (Ashok 2007). This choice is of course reasonable if one is looking for potential sites where wind/PV hybrid systems are feasible. However, in the context of electricity supply for recovery and reconstruction after natural disasters, it is not appropriate.

2. Some studies evaluate sites at higher latitude, where solar irradiation is very low during the whole winter season.

3. The cost of PV systems has been strongly reduced in the last few years. Some studies do not take into account economic considerations but rather make the comparison on an installed power basis.

\section{Sensitivity analysis}

To evaluate the robustness of the simulation results, sensitivity analyses have been performed. The larger load (20 kW peak) was analysed for the three different topologies: wind/battery, PV/battery and wind/PV/battery (where wind speed could come either from the ARMA or MERRA model).

1. LOLP $_{\max } 1 \%$ or $10 \%$.

2. Cost of WEC of $€ 3 / \mathrm{W}$.

3. Battery cost $€ 50 / \mathrm{kWh}$.

4. Winter peak in load.

Naturally a lower tolerated loss of load probability (compared with the default value 3\%) increases the system cost. With $\mathrm{LOLP}_{\max }$ set to $1 \%$, the costs increased on average by $14-19 \%$ for the different system topologies. For a few sites and topologies, the cost increased by more than $25 \%$. LOLP $_{\max }$ of $10 \%$ reduced the cost on average by $20-25 \%$ (maximum $41 \%$ ). Generally wind/battery systems are affected by tolerated LOLP $_{\max }$ to a slightly higher degree than PV/battery systems. Assuming a cost of WEC of $€ 3 \mathrm{~W}^{-1}$ reduced the average cost of wind/battery systems from $27 \%$ to $34 \%$. With ARMA wind at Cape Town or MERRA wind at Tbilisi, the cost of the wind/battery system became lower than the corresponding PV/battery system. The median cost of the wind/battery system was however still several times higher than the PV/battery system and most optimized hybrid systems contain little or no wind power. Halving the battery cost to $€ 50 / \mathrm{kWh}$ reduced the average system costs by $8-12 \%$. Slightly larger reductions 
were seen for the wind/battery system than the PV/battery system. Finally, using a winter peak load had a low impact on the results. The mean absolute difference in cost for the different types of systems was in the range of $3-4 \%$ and the offset was $+0-2 \%$.

\section{Conclusions}

The investment cost per yearly produced $\mathrm{kWh}$ and for an optimized energy system supplying small loads ( 2 or $20 \mathrm{~kW}$ peak) have been evaluated for 32 sites, predominantly in Africa and the Middle East. The sites represent all foreign activities of MSB at the end of 2012 and have thus not been chosen based on their wind or solar resource potential.

Because of sometimes massive amounts of missing wind speed data, ARMA models were used to generate time series with similar characteristics as the original series. It is shown that this methodology is successful even when data availability is very low. However, the cost of an optimized standalone energy system is underestimated when using ARMA data (bias $-11 \%$ ). Furthermore it is shown that when it is not possible to train an ARMA model (e.g. when measurements are only performed every $3 \mathrm{~h}$ ) a robust method is to "borrow" an ARMA model from a station with similar ACF and then back-transform the data to wind speed using parameters from the original time series.

For the evaluated sites, small-scale wind power is not able to match PV; the cost per produced $\mathrm{kWh}$ is higher, the risk for extended periods with very little production is higher and the cost of an optimized standalone system including batteries is substantially higher. For a few sites, the cost of a hybrid system containing WEC, PV and batteries is slightly lower than the system without WEC. For most sites, however, the optimized hybrid system contains virtually no wind power. The sensitivity analysis indicates that the results are robust.

The conclusion is not that small-scale wind power is not worth considering for off-grid applications. At remote sites with good wind conditions and/or strong seasonality in solar irradiation, a hybrid system with substantial installed wind power capacity can be optimal. A relatively high hub height and a large rotor compared with the rated power is probably preferable. For MSB foreign operations, often at low latitude and with little time for a thorough wind analysis, a
PV/battery system should be the first candidate when looking for alternatives or a system complementary to conventional diesel generators.

\section{Acknowledgements}

The study was funded by the Swedish Civil Contingencies Agency (MSB) through contract 20102787.

Jon Olauson, Anders Goude \& Mikael Bergkvist, Ångströmlaboratoriet, Lägerhyddsvägen 1, Box 534, 75121 Uppsala, Sweden

Email: jon.olauson@angstrom.uu.se

\section{References}

Albrecht, P.F., Bhavaraju, M.P., Biggerstaff, B.E., Billinton, R., Elsoe Jorgensen, G., Reppen N.D. and Shortley, P.B., 1979. IEEE reliability test system. IEEE Transactions on Power Apparatus and Systems, PAS-98, 2047-2054. doi:10.1109/TPAS.1979.319398

Ashok, S., 2007. Optimised model for community-based hybrid energy system. Renewable Energy, 32, 11551164. doi:10.1016/j.renene.2006.04.008

Bagen and Billinton, R., 2005. Evaluation of different operating strategies in small stand-alone power systems. IEEE Transactions on Energy Conversion, 20, 654-660. doi:10.1109/TEC.2005.847996

Billinton, R., Bagen and Cui, Y., 2003. Reliability evaluation of small stand-alone wind energy conversion systems using a time series simulation model. IEE Proceedings. Generation, Transmission and Distribution, 150, 96-100. doi:10.1049/ip-gid:20030068

Billinton, R., Chen H. and Ghajar, R., 1996. Time-series models for reliability evaluation of power systems including wind energy. Microelectronics Reliability, 36, 1253-1261. doi:10.1016/0026-2714(95)00154-9

Chen, P., Pedersen, T., Bak-Jensen, B. and Chen, Z., 2010. ARIMA-based time series model of stochastic wind power generation. IEEE Transactions on Power Systems, 25, 667-676. doi:10.1109/TPWRS.2009.2033277

Cryer, J.D. and Chan, K.-S., 2008. Time Series Analysis with Applications in $R$. Springer, New York.

Espinar, B., Blanc, P., Wald, L., Gschwind, B., Ménard, L., Wey, E., Thomas, C. and Saboret, L., 2012. HelioClim-3: a near-real time and long-term surface solar irradiance database. In: Workshop on "Remote Sensing Measurements for Renewable Energy”, Risoe: Denmark (2012). Roskilde, Denmark.

Huld, T., Gottschalg, R., Beyer, H.G. and Topič, M., 2010. Mapping the performance of PV modules, effects of module type and data averaging. Solar Energy, 84, 324338. doi:10.1016/j.solener.2009.12.002

Li, X. and Wang, H., 2012. Stochastic time series reconstruction of future wind farm output. In: 2012 IEEE Innovative Smart Grid Technologies - Asia (ISGT Asia), IEEE. 1-5. doi:10.1109/ISGT-Asia.2012.6303195

Lojowska, A., Kurowicka, D., Papaefthymiou, G. and Van der Sluis, L., 2010. Advantages of ARMA-GARCH wind speed time series modeling. In: 2010 IEEE 11th 
International Conference on Probabilistic Methods Applied to Power Systems (PMAPS), IEEE. 83-88. doi:10.1109/PMAPS.2010.5528979

Lundsager, P., Bindner, H., Clausen, N.-E., Frandsen, S., Hansen, L.H. and Hansen, J.C., 2001. Isolated Systems with Wind Power - Main Report. Ris $\emptyset$ National Laboratory, Roskilde, Denmark.

McWilliams, B. and Sprevak, D., 1982. Simulation of hourly wind speed and direction. Mathematics and Computers in Simulation, 24, 54-59.

Nema, P., Nema, R.K. and Rangnekar, S., 2009. A current and future state of art development of hybrid energy system using wind and PV-solar: a review. Renewable and Sustainable Energy Reviews, 13, 2096-2103. doi:10.1016/j.rser.2008.10.006

Pickard, W.F., 2012. A nation-sized battery? Energy Policy, 45, 263-267. doi:10.1016/j.enpol.2012.02.027

Pinson, P. and Madsen, H., 2012. Adaptive modelling and forecasting of offshore wind power fluctuations with Markov-switching autoregressive models. Journal of Forecasting, 31, 281-313. doi:10.1002/for.1194

Shanno, D.F., 1970, Conditioning of quasi-Newton methods for function minimization. Mathematics of Computation, 24, 647-656.

Shi, J., Qu, X. and Zeng, S., 2011. Short-term wind power generation forecasting: direct versus indirect Arimabased approaches. International Journal of Green Energy, 8, 100-112. doi:10.1080/15435075.2011 .546755

Stanton, A.F., Bleil, R.E. and Kais, S., 1997, A new approach to global minimization. Journal of Computational Chemistry, 18, 594-599. doi:10.1002/(SICI)1096987X(199703)18:4<594::AID-JCC12>3.0.CO;2-G
Thøgersen, M.L., Motta, M., Sørensen, T. and Nielsen, P., 2007. Measure-correlate-predict methods: case studies and software implementation. In: EWEC 2007 Scientific Proceedings. European Wind Energy Association, Brussels.

Tina, G., Gagliano, S. and Raiti, S., 2006. Hybrid solar/wind power system probabilistic modelling for long-term performance assessment. Solar Energy, 80, 578-588. doi:10.1016/j.solener.2005.03.013

WMO, 2012. Guide to Meteorological Instruments and Methods of Observation, 2008 Edition, updated in 2010. World Meteorological Organization, Geneva.

Xu, L., Ruan, X., Mao, C., Zhang, B. and Luo, Y., 2013. An improved optimal sizing method for wind-solar-battery hybrid power system. IEEE Transactions on Sustainable Energy, 4, 774-785. doi:10.1109/TSTE.2012.2228509

Yang, H., Zhou, W., Lu, L. and Fang, Z., 2008. Optimal sizing method for stand-alone hybrid solar-wind system with LPSP technology by using genetic algorithm. Solar Energy, 82, 354-367. doi:10.1016/j.solener.2007 .08 .005

Zhou, C., Qian, K., Allan, M. and Zhou, W., 2011. Modeling of the cost of EV battery wear due to V2G application in power systems. IEEE Transactions on Energy Conversion, 26, 1041-1050. doi:10.1109/TEC.2011.2159977

Zhou, W., Lou, C., Li, Z., Lu, L. and Yang, H., 2010. Current status of research on optimum sizing of stand-alone hybrid solar-wind power generation systems. Applied Energy, 87, 380-389. doi:10.1016/j.apenergy.2009 .08 .012

Manuscript Received 31 Jan., 2014, revised and accepted 5 Jun., 2014 